\title{
Qualidade de vida em mulheres no climatério atendidas na Atenção Primária
}

\author{
Quality of life of postmenopausal women attended at Primary Health Care \\ Calidad de vida de las mujeres en climaterio tratadas en la Atención Primaria
}

\begin{abstract}
Jéssica Steffany Miranda', Maria de Lourdes da Silva Marques Ferreira', José Eduardo Corrente"
'Universidade Estadual Paulista Júlio de Mesquita Filho, Faculdade de Medicina, Departamento de Enfermagem, Programa de Pós-Graduação em Enfermagem. Botucatu-SP, Brasil.

"Universidade Estadual Paulista Júlio de Mesquita Filho, Faculdade de Medicina, Instituto de Biociências, Departamento de Bioestatística. Botucatu-SP, Brasil.
\end{abstract}

\author{
Submissão: 18-12-2012 Aprovação: 17-07-2014
}

\section{RESUMO}

A vivência do climatério está cada vez mais presente, e demanda estratégias que melhorem a qualidade de vida das mulheres nesse período. A pesquisa objetivou avaliar a qualidade de vida de mulheres na fase do climatério, com ou sem uso da terapia de reposição hormonal (TRH). Estudo epidemiológico longitudinal, com amostra de 99 mulheres para cada grupo. Avaliaram-se as características sociodemográficas, clínicas e comportamentais. Foram aplicados à amostra a Menopause Rating Scale (MRS) e o Medical Outcomes Study 36-item Short-Form Health Survey (SF-36). Utilizaram-se os testes t de student, Qui-quadrado e Tukey. As usuárias de TRH apresentaram média etária de 50,76 $\pm 3,63$ anos, e as não usuárias de 48,95 \pm 6,27anos $(p=0,01)$. Relataram maior frequência de sintomas climatéricos de intensidade leve a moderada. Os aspectos sociais apresentaram escore abaixo de 50 para os dois grupos. Houve diferenças entre os grupos em relação aos componentes do SF-36 e MRS para estado geral de saúde, capacidade funcional, menor capacidade, depressão, insônia e fenômenos vasomotores.

Descritores: Qualidade de Vida; Climatério; Atenção Primária.

\section{ABSTRACT}

The experience of menopause is increasingly present, and demand strategies to improve the quality of life of women during this period. This research aimed to evaluate the quality of life for women in the climacteric phase, with or without the use of hormone replacement therapy (HRT). This is a longitudinal epidemiological study of a sample of 99 women per group. It was evaluated the sociodemographic, clinical and behavioral characteristics. It was used the Menopause Rating Scale (MRS) and the Medical Outcomes Study 36-item Short-Form Health Survey (SF-36). For data analysis, it was used the Student t test, chi-square and Tukey. HRT users had an average age of $50.76 \pm 3.63$ years, and nonusers of $48.95 \pm 6,27$ anos $(p=0.01)$. It was identified a higher frequency of moderate climacteric symptoms of mild intensity. The social aspects evidenced scores below 50 for the two groups. There were differences between groups with respect to the components of the SF-36 and MRS to general health, functional capacity, lower capacity, depression, insomnia and vasomotor phenomena.

Key words: Quality of Life; Climateric; Primary Health Care.

\section{RESUMEN}

La experiencia de la menopausia es cada vez más presente, demandando estrategias para mejorar la calidad de vida de las mujeres durante este periodo. Esa investigación tuvo como objetivo evaluar la calidad de vida de las mujeres en la fase climatérica, con o sin el uso de la terapia de reemplazo hormonal (TRH). Trata-se dé un estudio epidemiológico longitudinal, con una muestra de 99 mujeres por grupo. Fueran evaluadas las características sociodemográficas, clínicas y de comportamiento. Fueran aplicados a la muestra el Menopause Rating Scale (MRS) e el Medical Outcomes Study 36-item Short-Form Health Survey (SF-36). Utilizaran-se los testes t de Student, Qui-quadrado e Tukey. Las usuarias de TRH tenían una edad media de 50,76 \pm 3,63 años, y las no usuarias de $48,95 \pm 6,27$ anos $(p=0,01)$. Fue reportada una mayor frecuencia de síntomas climatéricos intensidad leve o moderada. Los aspectos sociales tuvieran puntuaciones por debajo de 50 para los dos grupos. Hubo diferencias entre los grupos con respecto a los componentes del SF-36 y el MRS para la salud en general, la capacidad funcional, la capacidad más baja, la depresión, el insomnio y los fenómenos vasomotores.

Palabras clave: Calidad de Vida; Climaterio; Atención Primaria de Salud. 


\section{INTRODUÇÃO}

A população feminina brasileira totaliza mais de $98 \mathrm{mi}-$ Ihões de mulheres. São as principais usuárias do Sistema Único de Saúde (SUS), seja para o seu próprio atendimento ou acompanhando seus familiares, representando 50,77\% da população brasileira(1). Por isso, a vivência do climatério pelas mulheres está cada vez mais presente, e demanda estratégias que melhorem a qualidade de vida nesse período.

A atenção integral à saúde da mulher pressupõe assistência em todas as fases de sua vida. O climatério, por compreender um período relativamente longo da vida da mulher, deve merecer atenção crescente da sociedade, pois a expectativa de vida após a menopausa é atualmente equivalente ao período de vida reprodutiva ${ }^{(2)}$. Assim, considera-se o climatério como uma fase da vida biológica da mulher que representa a transição entre o período reprodutivo e o não reprodutivo(3) e não um processo patológico ${ }^{(4)}$. O período etário aceito em que a mulher poderá experimentar os sintomas associados ao climatério é a partir dos 40 anos.

Nas últimas décadas há referências da universalidade dos sintomas climatéricos, apesar deles sofrerem influências de características sociodemográficas, como por exemplo, a raça. A transição climatérica é um fenômeno cultural extremamente variável e a complexidade dos fatores hormonais e psicossocioculturais e o próprio envelhecimento biológico produzem uma grande variabilidade de sintomas como também consequências para a saúde em longo prazo ${ }^{(5)}$.

O diagnóstico do climatério é dominantemente clínico, baseado na faixa etária, no padrão menstrual alterado e manifestações climatéricas. No entanto, para avaliação quantitativa do climatério, alguns índices, denominados de índices menopausais, foram criados e todos têm como princípio a somatória ponderal das manifestações climatéricas; também são utilizados para a realização de protocolos de pesquisa para comparação de tratamentos ${ }^{(6)}$.

Nos últimos anos, têm surgido indagações sobre se os sintomas climatéricos e a tendência ao comprometimento intenso ou não da qualidade de vida no climatério, além do hipoestrogenismo, se não estariam associados também a fatores psicossociais e culturais relacionados ao processo de envelhecimento ou, então, pela interação destes ${ }^{(7)}$. Atualmente a hipótese é de que qualidade de vida no climatério seria influenciada tanto pela presença dos sintomas decorrentes do declínio estrogênico, como por fatores psicossociais e culturais ligados ao próprio processo de envelhecimento ${ }^{(7-8)}$.

Frente à complexidade da síndrome climatérica e dos seus possíveis reflexos na qualidade de vida feminina, tem sido proposta uma nova abordagem, destacando a importância de uma escuta qualificada paralela às intervenções clínicas necessárias, de forma a permitir maior compreensão do processo crítico existencial envolvido, onde aspectos psicológicos relacionados ao envelhecer se mesclam com aqueles resultantes do esgotamento hormonal(8-9).

Desta forma, de fato, com o que se relacionada a qualidade de vida de mulheres que passam pelo período do climatério? Portanto, a presente pesquisa teve por objetivo avaliar a qualidade de vida de mulheres na fase do climatério atendidas na atenção primária à saúde.

\section{METODOLOGIA}

Trata-se de uma pesquisa epidemiológica, prospectiva, longitudinal. Neste tipo de investigação, procura-se verificar a frequência de um determinado agravo na presença ou ausência de um determinado fator condicionante/determinante (exposição). Neste caso em especial, procura-se saber se há modificações na qualidade de vida de mulheres submetidas à TRH e não submetidas à TRH. É procurado, então, esclarecer uma dada associação entre uma exposição, em particular, e um efeito específico.

Participaram mulheres no período do climatério escolhidas aleatoriamente (sorteio realizado com os prontuários da faixa etária visada), dos serviços de atenção primária à saúde de um município do interior paulista. As participantes selecionadas foram divididas em dois grupos: desejosas de realizar a Terapia de Reposição Hormonal (TRH) e não desejosas de realizar a $\mathrm{TRH}$, de acordo com os critérios de inclusão: mulheres com idade entre 40 e 65 anos de idade e menopausa há no máximo 05 ano; e exclusão: mulheres que apresentem doença psiquiátrica; que estivessem usando medicamentos psiquiátricos ou outro tipo de medicação que pudesse interferir em seu estado de consciência; que fizessem uso de qualquer tipo de droga ou uso abusivo de álcool; que já iniciaram a TRH; que fizeram uso de qualquer medicamento não hormonal para alívio dos sintomas associados ao climatério no último ano e fatores que contraindicassem absolutamente a TRH).

Para a definição da amostra utilizou-se o número de prontuários de mulheres na faixa etária de 40 a 65 anos (5.000), segundo levantamento na Unidade onde é realizado o atendimento de ginecologia do município. Considerando que a prevalência de boa qualidade de vida em mulheres no período do climatério seja de $50 \%$, ou seja, uma prevalência desconhecida, uma margem de erro de $10 \%$ e coeficiente de confiança de $95 \%$, o tamanho amostral mínimo foi de 96 pacientes avaliadas antes e após a TRH. Para o grupo controle, teve-se o mesmo valor de "n". Foram voluntárias 99 mulheres para cada grupo. A faixa etária escoIhida deu-se em concordância com o Consenso Brasileiro Multidisciplinar da Sociedade Brasileira de Climatério - SOBRAC ${ }^{(8)}$.

As mulheres selecionadas para o estudo foram acompanhadas pelos ginecologistas do município, com termo firmado de colaboração com os mesmos. As mulheres em uso de TRH foram avaliadas no início do estudo e após o término do tratamento, conforme critério médico (sendo o período máximo de 06 meses); as não usuárias de TRH foram avaliadas no início e depois de 06 meses, sendo a avaliação de no mínimo 03 meses.

O período de coleta de dados deu-se entre novembro de 2011 e maio de 2012. As entrevistas foram realizadas no local de maior comodidade para a entrevistada, sendo nas Unidades Básicas do município e no domicílio, inclusive. As entrevistas duraram em média 25 (vinte e cinco) minutos e foram feitas por apenas um dos pesquisadores. Os dados foram tabulados em dupla inserção pelos pesquisadores para garantir a fidedignidade dos mesmos. 
Foram utilizados três instrumentos para atender aos objetivos da presente pesquisa. Para identificação e caracterização da usuária, o pesquisador criou um formulário de entrevista, sendo este um instrumento de identificação com dados clínicos, comportamentais e sociodemográficos. Para a avaliação da qualidade de vida de forma genérica e abrangente, optou-se pelo instrumento validado denominado Medical Outcomes Study 36-item Short-Form Health Survey (SF-36). O SF-36, utilizado para avaliação da saúde geral, atualmente é um dos instrumentos mais conhecidos e difundidos na área de saúde ${ }^{(10)}$, já traduzido e validado no Brasil ${ }^{(11)}$. É um questionário multidimensional, composto por 11 questões e 36 itens, com oito componentes ou domínios: capacidade funcional (10 itens), aspectos físicos (4 itens), dor (2 itens), estado geral de saúde (5 itens), vitalidade (4 itens), aspectos sociais (2 itens), aspectos emocionais (3 itens), saúde mental (5 itens). Cada componente do SF-36 corresponde a um valor, que varia de zero a 100, onde zero corresponde ao pior e 100 ao melhor estado de saúde, derivados de cálculos correlacionais entre as respostas dos domínios.

Para a avaliação dos sintomas em específico, a escala de maior viabilidade e complementação do SF-36 foi a Escala de Avaliação da Menopausa - Menopause Rating Scale (MRS), por ser rápida na aplicação e bastante direta e específica quanto à validação dos dados. A Escala de Avaliação da Menopausa (MRS) é um instrumento que tem em vista a intensidade da sintomatologia do climatério. É considerada uma ferramenta valiosa na avaliação dos sintomas mais prevalentes para esta fase da vida feminina. Esta escala foi padronizada, inicialmente, na Alemanha por Heinemann et al. ${ }^{(12)}$, tendo sido traduzida para vários idiomas, inclusive o português. A validação para o Brasil foi realizada em 2002, pelo Instituto Internacional "NFO", com sede nos Estados Unidos ${ }^{(13)}$.

Foram assegurados sigilo e seguimento do tratamento para todas as mulheres que utilizaram a $\mathrm{TRH}$, sem prejuízo da assistência após o término da pesquisa. No que tange ao tratamento da menopausa, o protocolo da instituição escolhida, era composto por isoflavona de soja, estrógeno equino conjugado e estradiol mais acetato de noretisterona.

Faz-se necessário salientar que, diante da escolha aleatória da amostra, 20 mulheres recusaram-se a participar do estudo ou foram perdidas no decorrer do acompanhamento, tendo como justificativa para tal a insatisfação em responder aos questionários e não verificação da relevância de sua participação.

A pesquisa foi aprovada pelo Comitê de Ética em Pesquisa da Faculdade de Medicina de Botucatu-Unesp, protocolo CEP 3948-2011, de 01 de Agosto de 2011, em respeito à Resolução 466 de 2012 do Conselho Nacional de Saúde.

Para os componentes do SF-36, os mesmos foram analisados pela média, desvio padrão e percentil, após cálculos dos domínios. Foi utilizado o Teste de Tukey para comparação entre os grupos e teste do Qui-quadrado ou exato de Fisher para diferenças entre proporções. As comparações foram consideradas estatisticamente significativas com $p<0,05$.

\section{RESULTADOS E DISCUSSÃO}

As usuárias de TRH apresentaram média etária de 50,76 \pm 3,63 anos e as não usuárias de 48,95 $\pm 6,27$ anos $(p=0,014)$. Na variável nível de escolaridade, observamos que o grupo intervenção tem maior proporção de mulheres com ensino superior, dando significância nesta diferença. Ainda, quanto à renda própria, as diferenças não se mostraram estatisticamente significativas (Tabela 01).

Quando as mulheres foram perguntadas a respeito das comorbidades (Tabela 02), evidenciou-se significativa prevalência de HAS e Artralgia, com cerca de 20 e 30\% de distribuição nos grupos. Porém, em comparação entre os grupos, não houve diferença estatisticamente significativa. Apenas para ingestão de álcool e tabagismo observou-se diferença. O uso eventual de álcool no grupo intervenção é maior, ao passo que existem mais não tabagistas e ex-tabagistas no grupo controle.

Tabela 1 - Caracterização dos grupos segundo cor, nível de escolaridade e renda própria

\begin{tabular}{|c|c|c|c|c|c|}
\hline \multirow[t]{2}{*}{ Variável } & \multicolumn{2}{|c|}{ Grupo Controle $(n=99)$} & \multicolumn{2}{|c|}{ Grupo Intervenção $(\mathbf{n}=99)$} & \multirow[b]{2}{*}{$\mathbf{p}^{*}$} \\
\hline & Frequência & Percentual & Frequência & Percentual & \\
\hline Cor & & & & & 0,356 \\
\hline Branca & 66 & 66,67 & 73 & 73,74 & \\
\hline Preta & 09 & 9,09 & 11 & 11,11 & \\
\hline Parda & 23 & 23,23 & 15 & 15,15 & \\
\hline Amarela & 01 & 1,01 & 0 & 0,00 & \\
\hline Nível de Escolaridade & & & & & 0,005 \\
\hline Ensino Fundamental & 58 & 58,59 & 42 & 42,42 & \\
\hline Ensino Médio & 31 & 31,31 & 27 & 27,27 & \\
\hline Ensino Superior & 08 & 8,08 & 30 & 30,30 & \\
\hline Renda própria & & & & & 0,106 \\
\hline 01 salário mínimo & 37 & 37,37 & 21 & 21,21 & \\
\hline 02 salários mínimos & 10 & 10,10 & 14 & 14,14 & \\
\hline 03 salários mínimos & 09 & 9,09 & 10 & 10,10 & \\
\hline Mais de 03 salários mínimos & 01 & 1,01 & 04 & 4,04 & \\
\hline Não tinham renda própria & 42 & 42,42 & 50 & 50,51 & \\
\hline
\end{tabular}

*Teste Qui-quadrado 
Tabela 2 - Distribuição das comorbidades nos grupos estudados

\begin{tabular}{|c|c|c|c|c|c|}
\hline \multirow{2}{*}{ Variável } & \multicolumn{2}{|c|}{$\begin{array}{l}\text { Grupo Controle } \\
\qquad(\mathrm{n}=99)\end{array}$} & \multicolumn{2}{|c|}{$\begin{array}{l}\text { Grupo Intervenção } \\
\qquad(\mathrm{n}=99)\end{array}$} & \multirow[b]{2}{*}{$\mathbf{p}^{*}$} \\
\hline & Frequência & Percentual & Frequência & Percentual & \\
\hline HAS & & & & & 0,143 \\
\hline Sim & 43 & 43,43 & 33 & 33,33 & \\
\hline Não & 56 & 56,57 & 66 & 66,67 & \\
\hline DM & & & & & 0,788 \\
\hline Sim & 8 & 8,08 & 7 & 7,07 & \\
\hline Não & 91 & 91,92 & 92 & 92,93 & \\
\hline Artralgia & & & & & 0,101 \\
\hline Sim & 30 & 30,30 & 20 & 20,20 & \\
\hline Não & 69 & 69,70 & 79 & 79,80 & \\
\hline Osteoporose & & & & & 0,091 \\
\hline Sim & 13 & 13,13 & 6 & 6,06 & \\
\hline Não & 86 & 86,87 & 93 & 93,94 & \\
\hline
\end{tabular}

*Teste Qui-quadrado

Para o estado marital, há diferença entre os grupos ( $p$ $<0,001)$; o grupo controle tem maior frequência de mulheres sem companheiro $(26,26 \%)$ e sem parceiro sexual $(29,29)$; já o grupo intervenção apresentou maior proporção de mulheres com parceiro sexual e companheiro $(89,90 \%)$.

Em relação aos hábitos de vida e presença de dificuldades sexuais, conjugais, familiares e conjugais, houve diferença estatística entre os grupos, excetuando-se as dificuldades artísticas e atividades religiosas (Tabela 3 ).

Em relação à idade da menarca, não se observou diferença, estando a média das idades por volta dos 12 anos, com o grupo controle apresentando $12,45 \pm 1,75$ e o grupo intervenção $12,85 \pm 1,59(p=0,0738)$. Do mesmo modo, o tempo de menopausa tem diferença estatisticamente significativa nos grupos, com cerca de 2 anos - grupo controle com $1,58 \pm 2,0$ e grupo intervenção com 2,24 $\pm 1,90(p=0,014)$. Estatisticamente significativa foi a diferença entre a presença de fluxo menstrual, que é nula no grupo intervenção. Quando indagadas a respeito de quantos dias permanecia o fluxo menstrual, a média foi de $2,60+2,81$.

Quanto à cor/etnia não se observou a associação com a intensidade das ondas de calor, provavelmente pela dificuldade de avaliação dessa variável em nosso meio. Cabe ressaltar que, no presente estudo, essa variável foi classificada segundo autodeclaração da participante. Importante também destacar que o papel da cor/etnia nos níveis hormonais durante a transição climatérica é pouco conhecido. O estudo Study of Women's Health Across the Nation demonstrou que mulheres americanas afrodescendentes foram as que apresentaram os menores níveis de testosterona e de sulfato de deidroepiandrosterona quando comparadas às caucasianas, hispânicas e orientais. Possivelmente por este motivo, as afro-americanas foram as que reportaram maior prevalência de ondas de calor $^{(14)}$.
Na avaliação específica dos sintomas climatéricos com base no instrumento MRS, verificou-se que não há severidade dos sintomas, uma vez que todos os escores de avaliação não passaram de 0,40 para as queixas, excetuando-se os fenômenos vasomotores, que tiveram escores acima de 0,50 (Tabela 4).

Para os domínios do SF-36 observou-se uma boa qualidade de vida. Apenas o domínio de dor esteve com escores por volta de 50 e o domínio de aspectos sociais, abaixo de 50 .

As ondas de calor frequentemente determinam um impacto negativo na qualidade de vida, relacionando-se a alterações do sono com consequente fadiga, irritabilidade, desconforto físico e problemas no trabalho. No Brasil, segundo estudo de base populacional, a prevalência de ondas de calor é de $70,3 \%$ em mulheres no climatério. Embora pareça ter como principal causa a deficiência estrogênica, as ondas de calor apresentam prevalência e intensidade que variam segundo características da população estudada. Dessa forma, o índice de massa corpórea (IMC), tabagismo, consumo de álcool, escolaridade, antecedente de ooforectomia ou de câncer são alguns fatores associados à intensidade das ondas de calor ${ }^{(14)}$.

Segundo $\mathrm{O}^{\prime} \mathrm{dea}^{(15)}$, dentre os fatores associados à qualidade de vida da mulher ao longo do seu processo de envelhecimento, os mais relevantes são as suas condições físicas e emocionais prévias, a sua inserção social e experiências frente a eventos vitais. Mais recentemente, verificou-se que a sintomatologia climatérica e a qualidade de vida no climatério eram influenciadas também pelas atitudes e percepções das próprias mulheres em relação à menopausa, o que pode ter ocorrido no estudo de Silva-Filho e Costa ${ }^{(16)}$. Nesse contexto, a preocupação com a qualidade de vida se contrapõe com a tendência moderna de tecnologização excessiva da Medicina, contribuindo para que se resguarde um dos mais atuais paradigmas da saúde, a questão da humanização, o que vai ao encontro, inclusive, com o proposto pelos inúmeros protocolos e consensos em relação ao climatério.

Recentemente um estudo de coorte que avaliou o impacto da TRH na qualidade de vida de mulheres climatéricas verificou que após um ano do início dessa terapia houve melhora estatisticamente significativa das alterações do sono, capacidade funcional e dor, porém sem benefícios clínicos significativos avaliados ${ }^{(17)}$. Nesse mesmo estudo, após três anos de seguimento não foi observada melhora significativa em nenhum dos domínios da qualidade de vida.

$\mathrm{Na}$ presente pesquisa, observou-se que a TRH foi iniciada em média após dois anos de início da menopausa, o que pode justificar a melhora na qualidade de vida, ainda que discreta nas variáveis sintomas vasomotores, insônia, depressão e menor capacidade do MRS. Com relação aos domínios do SF-36, não houve melhora estatisticamente significativa após a intervenção.

Optamos por definir como critério de exclusão as mulheres que estivessem utilizando algum medicamente antidepressivo ou semelhante que pudesse interferir nas variáveis insônia e depressão, assim como as outras associadas, mas em estudo de inquérito populacional realizado no Brasil mostrou que os tranquilizantes/antidepressivos foram a medicação de escoIha $(28,3 \%)$ para mulheres que procuraram o serviço médico 
Tabela 3 - Distribuição nos grupos estudados dos hábitos de vida

\begin{tabular}{|c|c|c|c|c|c|}
\hline \multirow{2}{*}{ Variável } & \multicolumn{2}{|c|}{ Grupo Controle $(n=99)$} & \multicolumn{2}{|c|}{ Grupo Intervenção $(n=99)$} & \multirow[b]{2}{*}{$\mathbf{p}^{*}$} \\
\hline & Frequência & Percentual & Frequência & Percentual & \\
\hline Ingestão de álcool & & & & & 0,002 \\
\hline Não faz uso & 87 & 87,88 & 70 & 70,71 & \\
\hline Diariamente & 0 & & 0 & & \\
\hline Eventualmente & 12 & 12,12 & 29 & 29,29 & \\
\hline Tabagismo & & & & & $<0,001$ \\
\hline Não tabagista & 84 & 84,85 & 68 & 68,69 & \\
\hline Tabagista & 12 & 12,12 & 15 & 15,15 & \\
\hline Ex-tabagista & 3 & 3,03 & 16 & 16,16 & \\
\hline Atividades esportivas & & & & & $<0,001$ \\
\hline Sim & 26 & 26,26 & 30 & 30,30 & \\
\hline Não & 73 & 73,74 & 69 & 69,70 & \\
\hline Atividades artísticas & & & & & 0,281 \\
\hline Sim & 34 & 34,34 & 27 & 27,27 & \\
\hline Não & 65 & 65,66 & 72 & 72,73 & \\
\hline Atividades religiosas & & & & & 0,296 \\
\hline Sim & 75 & 75,76 & 81 & 81,82 & \\
\hline Não & 24 & 24,24 & 18 & 18,18 & \\
\hline Dificuldades sexuais & & & & & 0,005 \\
\hline Sim & 12 & 12,12 & 2 & 2,02 & \\
\hline Não & 87 & 87,88 & 97 & 97,98 & \\
\hline Dificuldades conjugais & & & & & 0,003 \\
\hline Sim & 8 & 8,08 & 0 & 0,00 & \\
\hline Não & 91 & 91,92 & 99 & 100,00 & \\
\hline Dificuldades familiares & & & & & 0,002 \\
\hline Sim & 9 & 9,09 & 0 & 0,00 & \\
\hline Não & 90 & 90,91 & 99 & 100,00 & \\
\hline Dificuldades sociais & & & & & 0,003 \\
\hline Sim & 8 & 8,08 & 0 & 0,00 & \\
\hline Não & 91 & 91,92 & 99 & 100,00 & \\
\hline Alimentação diária & & & & & $<0,001$ \\
\hline 1 & 1 & 1,01 & 2 & 2,02 & \\
\hline 2 & 27 & 27,27 & 2 & 2,02 & \\
\hline 3 & 26 & 26,26 & 40 & 40,40 & \\
\hline 4 ou mais & 45 & 45,45 & 55 & 55,56 & \\
\hline
\end{tabular}

* Teste Qui-quadrado

por queixas climatéricas ${ }^{(18)}$. Em geral, os tranquilizantes são usados para alívio do nervosismo, ansiedade, irritabilidade e insônia. Próximo ao período da menopausa, essas queixas relacionadas ao humor podem estar associadas ao climatério. Isso sugere que a TRH pode aliviar as queixas relacionadas ao humor na mulher climatérica ${ }^{(18)}$.

Esse efeito benéfico da TRH sobre o estado do humor parece não só ser decorrente do alívio dos sintomas vasomotores, mas também pode estar relacionado a um efeito direto dos hormônios sexuais femininos sobre o sistema nervoso central. Isso leva a crer que mulheres em uso de tranquilizantes / antidepressivos poderiam ser beneficiadas com a TRH.
Especula-se que a alta taxa de uso de tranquilizante em mulheres que consultam um médico por queixas climatéricas possa, em alguns casos, representar um diagnóstico errôneo em relação aos sintomas psicológicos, sem que se correlacionasse a presença desses sintomas com o estado menopausal ${ }^{(19)}$.

Isso poderia explicar o porquê dos tranquilizantes terem sido prescritos no lugar da TRH. Uma outra explicação poderia ser a falta de familiaridade, conhecimento ou insegurança por parte dos médicos a respeito da terapia de reposição hormonal e mesmo a falta de informação sobre os outros efeitos benéficos da TRH em longo prazo.

$\mathrm{Na}$ presente pesquisa, as participantes eram usuárias da atenção básica e, portanto, pertencentes a estratos sociais menos favorecidos e de baixa escolaridade, em sua maioria. Apesar disso, podemos considerar de uma maneira geral que a qualidade de vida dessas mulheres foi boa, independentemente do uso ou não da terapia hormonal, uma vez que dos oito componentes avaliados no SF-36, apenas aspectos sociais apresentou escore abaixo de 50, o que pode ser justificado, em partes, pelo estrato social a que pertencem. $\mathrm{O}$ fato dessas mulheres terem acesso a serviços de saúde especializados no atendimento e, sobretudo, no entendimento da mulher climatérica pode minimizar os efeitos da terapia hormonal sobre a qualidade de vida. A consulta com profissionais especializados pode exercer um efeito positivo na percepção da qualidade de vida. Cabe evidenciar que profissional especializado é aquele capacitado para o manejo da saúde da mulher neste período, podendo ou não estar alocado em ambulatórios de especialidade, justificando que a atenção básica pode fornecer tratamento especializado à medida que está capacitada para tal dentro da rede de atenção à saúde.

Outro ponto que merece atenção foi a prevalência de comorbidades, sobretudo em não usuárias de TRH, que provavelmente associou-se à prescrição de alguma medicação, que pode ter exercido algum efeito placebo nesse grupo que não conseguimos mensurar, assim como a automedicação, principalmente a analgésica. 
Tabela 4 - Qualidade de vida geral e específica para os sintomas climatéricos nos grupos estudados nos tempos 0 e 6

\begin{tabular}{|c|c|c|c|c|c|c|c|c|}
\hline \multirow{3}{*}{ Variáveis } & \multicolumn{3}{|c|}{ Grupo Controle $(\mathrm{n}=99)$} & \multicolumn{5}{|c|}{ Grupo Intervenção $(\mathrm{n}=99)$} \\
\hline & \multicolumn{3}{|c|}{ Média e Desvio Padrão } & \multicolumn{5}{|c|}{ Média e Desvio Padrão } \\
\hline & Tempo 0 & p* & Tempo 6 & $\mathbf{p}^{*}$ & Tempo 0 & $\mathbf{p}^{*}$ & Tempo 6 & $\mathbf{p}^{*}$ \\
\hline \multicolumn{9}{|l|}{ MRS } \\
\hline Fenômenos Vasomotores & $0,27 \pm 0,34$ & $\mathrm{aA}$ & $0,31 \pm 0,51$ & $\mathrm{aA}$ & $0,52 \pm 0,38$ & $\mathrm{aB}$ & $0,41 \pm 0,39$ & $\mathrm{aA}$ \\
\hline Queixas Cardíacas & $0,21 \pm 0,30$ & $\mathrm{aA}$ & $0,22 \pm 0,31$ & $\mathrm{aA}$ & $0,15 \pm 0,26$ & $\mathrm{aA}$ & $0,12 \pm 0,23$ & $\mathrm{aA}$ \\
\hline Insônia & $0,27 \pm 0,34$ & $\mathrm{aA}$ & $0,26 \pm 0,35$ & $\mathrm{aA}$ & $0,18 \pm 0,30$ & $\mathrm{aA}$ & $0,14 \pm 0,26$ & $\mathrm{aB}$ \\
\hline Depressão & $0,25 \pm 0,34$ & $\mathrm{aA}$ & $0,26 \pm 0,36$ & $\mathrm{aA}$ & $0,15 \pm 0,26$ & $\mathrm{aA}$ & $0,12 \pm 0,23$ & $\mathrm{aB}$ \\
\hline Irritabilidade & $0,24 \pm 0,32$ & $\mathrm{aA}$ & $0,35 \pm 0,35$ & $\mathrm{aA}$ & $0,35 \pm 0,33$ & $\mathrm{aA}$ & $0,31 \pm 0,32$ & $\mathrm{aA}$ \\
\hline Menor Capacidade & $0,28 \pm 0,33$ & $\mathrm{aA}$ & $0,28 \pm 0,35$ & $\mathrm{aA}$ & $0,15 \pm 0,24$ & $\mathrm{aB}$ & $0,14 \pm 0,22$ & $\mathrm{aB}$ \\
\hline Sexualidade & $0,32 \pm 0,34$ & $\mathrm{aA}$ & $0,29 \pm 0,34$ & $\mathrm{aA}$ & $0,36 \pm 0,33$ & $\mathrm{aA}$ & $0,34 \pm 0,33$ & $\mathrm{aA}$ \\
\hline Queixas Urinárias & $0,19 \pm 0,32$ & $\mathrm{aA}$ & $0,20 \pm 0,32$ & $\mathrm{aA}$ & $0,13 \pm 0,26$ & $\mathrm{aA}$ & $0,12 \pm 0,24$ & $\mathrm{aA}$ \\
\hline Secura Vaginal & $0,17 \pm 0,30$ & $\mathrm{aA}$ & $0,15 \pm 0,29$ & $\mathrm{aA}$ & $0,25 \pm 0,31$ & $\mathrm{aA}$ & $0,22 \pm 0,29$ & $\mathrm{aA}$ \\
\hline Queixas Locomotoras & $0,33 \pm 0,37$ & $\mathrm{aA}$ & $0,33 \pm 0,39$ & $\mathrm{aA}$ & $0,26 \pm 0,46$ & $\mathrm{aA}$ & $0,24 \pm 0,44$ & $\mathrm{aA}$ \\
\hline \multicolumn{9}{|l|}{ SF-36 } \\
\hline Capacidade Funcional & $77,22 \pm 26,13$ & $\mathrm{aA}$ & $76,21 \pm 25,91$ & $\mathrm{aA}$ & $93,13 \pm 9,76$ & $\mathrm{aB}$ & $93,13 \pm 9,76$ & $\mathrm{aB}$ \\
\hline Aspectos Físicos & $74,24 \pm 42,02$ & $\mathrm{aA}$ & $72,98 \pm 40,83$ & $\mathrm{aA}$ & $84,34 \pm 23,03$ & $\mathrm{aA}$ & $85,61 \pm 21,46$ & $\mathrm{aA}$ \\
\hline Dor & $53,74 \pm 9,75$ & $\mathrm{aA}$ & $53,74 \pm 9,75$ & $\mathrm{aA}$ & $56,97 \pm 10,05$ & $\mathrm{aA}$ & $56,97 \pm 10,05$ & $\mathrm{aA}$ \\
\hline Estado Geral de Saúde & $70,05 \pm 17,74$ & $\mathrm{aA}$ & $70,25 \pm 17,86$ & $\mathrm{aB}$ & $79,80 \pm 10,69$ & $\mathrm{aB}$ & $79,34 \pm 10,44$ & $\mathrm{aB}$ \\
\hline Vitalidade & $66,26 \pm 22,54$ & $\mathrm{aA}$ & $66,21 \pm 22,75$ & $\mathrm{aA}$ & $69,75 \pm 17,76$ & $\mathrm{aA}$ & $69,95 \pm 17,88$ & $\mathrm{aA}$ \\
\hline Aspectos Sociais & $43,73 \pm 10,00$ & $\mathrm{aA}$ & $47,47 \pm 10,10$ & $\mathrm{aA}$ & $46,09 \pm 12,45$ & $\mathrm{aA}$ & $46,34 \pm 12,28$ & $\mathrm{aA}$ \\
\hline Limitação por Aspectos Emocionais & $80,13 \pm 40,66$ & $\mathrm{aA}$ & $79,12 \pm 39,44$ & $\mathrm{aA}$ & $91,25 \pm 25,01$ & $\mathrm{aA}$ & $92,26 \pm 23,24$ & $\mathrm{aA}$ \\
\hline Saúde Mental & $72,36 \pm 21,21$ & $\mathrm{aA}$ & $71,72 \pm 21,33$ & $\mathrm{aA}$ & $73,45 \pm 18,19$ & $\mathrm{aA}$ & $73,49 \pm 18,21$ & $\mathrm{aA}$ \\
\hline
\end{tabular}

a: Comparação do tempo fixando o grupo; A: comparação dos grupos fixando o tempo

* Teste de Tukey ajustado

Os escores máximos foram observados nos componentes capacidade funcional, aspectos físicos e aspectos emocionais. O componente aspectos físicos avalia a interferência dos problemas de saúde na realização do trabalho e das atividades diárias, e cerca de metade dessas mulheres, em ambos os grupos, exercia atividades profissionais. Talvez não lhes seja possível apresentar limitações nesse componente. No componente emocional, que avalia a interferência de problemas emocionais nas atividades sociais, também se observou escore máximo. Esse fato pode significar que a menopausa não interferiu negativamente na integração social dessas mulheres. $\mathrm{O}$ menor escore foi observado nos aspectos sociais (por volta de 46) para ambos os grupos. Esse resultado pode ser explicado em razão do excesso de atividade dentro do lar associado com o trabalho, o que impossibilita maiores atividades sociais, além da baixa renda.

Ainda, é possível que a sobrecarga causada pela dupla jornada de trabalho seja responsável pelo comprometimento da vitalidade. Outros autores, aplicando o SF-36 em mulheres no climatério, também verificaram baixos escores no componente vitalidade ${ }^{(20)}$.

Zahar $^{(21)}$ sugere que estudos longitudinais poderão mostrar a mudança do escore de qualidade de vida a partir da prescrição da $\mathrm{TRH}$, enriquecendo as informações existentes até o momento e ampliando a compreensão dos fatores associados à qualidade de vida dessas mulheres. A utilização de uma amostra populacional permitiria inferências relativas às mulheres climatéricas de forma mais ampla e não apenas relativas àquelas de ambulatórios especializados. Neste estudo longitudinal, apesar das limitações de amostra e de ser realizado em um único município, pudemos observar que a TRH exerce pouca influência na modificação dos sintomas associados à percepção de qualidade de vida. De fato, qualidade de vida, corroborando com diversos autores, é muito mais do que sintomas clínicos, mas uma forma de viver e de encarar as adversidades colocadas nesta vivência em que a medicação de reposição hormonal pouco tem significância.

É importante se considerar a proposta de utilizar também métodos qualitativos nas pesquisas voltadas a avaliar a qualidade de vida no climatério, a despeito de a metodologia quantitativa ainda se mostrar hegemônica e predominante na literatura especializada, principalmente entre a classe médica. A metodologia qualitativa, quando adequadamente conduzida, permite que se mostrem com mais liberdade a subjetividade e a multidimensionalidade - tão importantes na aferição da qualidade de vida. Além disso, os defensores de enfoques qualitativos enfatizam que a utilização de medidas padronizadas pode levar a respostas estereotipadas, que muitas vezes têm pouco ou nenhum significado para a pessoa ${ }^{(21)}$.

\section{CONCLUSÕES}

Para esta pesquisa, conclui-se que a TRH tem efeitos significativos na amenização dos fenômenos vasomotores, o que 
pode ser observado na comparação intragrupo intervenção.

Estando a qualidade de vida mais ligada a fatores emocionais, psicológicos e sociais, as mulheres de ambos os grupos apresentaram qualidade de vida boa, sem diferença estatisticamente significativa para os escores, excetuando-se a capacidade funcional, os aspectos físicos e o estado geral de saúde do instrumento SF-36 e insônia, depressão e menor capacidade do instrumento SF-36 na comparação entre os grupos. Para o SF-36 após a TRH, não houve diferença estatisticamente significativa. Em ambos os grupos a qualidade de vida foi considerada boa, pois não existem escores abaixo de 50 para o SF-36, excetuando-se os aspectos sociais.

Sugere-se que os resultados obtidos neste estudo sejam utilizados como subsídios para estratégias na política de atenção à mulher, evidenciando as inserções de atividades de âmbito relacional e social, o que infere na significativa melhoria dos sintomas advindos da vivência e aceitação do período. Ainda, os dados servem para nortear as melhores práticas clínicas adotadas e devem incitar mais pesquisas em outras populações.

\section{REFERÊNCIAS}

1. Portal da Saúde. Saúde da mulher [Internet]. [S.I.]: Portal da Saúde; [s.d.] [acesso em 09 de setembro de 2013]. Disponível em: http://www.ses.se.gov.br

2. Fundação Seade [Internet]. [S.I.]: Fundação Seade; [s.d.] [acesso em 21 de junho de 2013]. Disponível em: http:// www.seade.sp.gov.br/produtos/projpop/index.php

3. Halbe HW, Fonseca AM. Síndrome do climatério. In: Halbe HW. Tratado de Ginecologia. 3. ed. São Paulo (SP): Roca; 2000. p. 1519-57.

4. Almeida $A B$. Reavaliando o climatério: enfoque atual e multidisciplinar. São Paulo (SP): Atheneu; 2003.

5. Pedro AO, Pinto-Neto AM, Costa-Paiva LHS, Osis MJD, Hardy EE. Sindorme do climatério: inquérito populacional domiciliar em Campinas, SP. Rev Saúde Pública. 2003;37(6):735-42.

6. Almeida AB. Reavaliando o climatério: Enfoque atual e multidisciplinar. São Paulo (SP): Atheneu; 2003.

7. Lorenzi DRS, Baracat EC, Saciloto B, Padilha Júnior I. Fatores associados à qualidade de vida após menopausa. Rev Assoc Med Bras [Internet]. 2006 [acesso em 23 de fevereiro de 2012];52(5):312-7. Disponível em: http://www.scielo.br/scielo.php?pid = S0104-42302006000500017\&script =sci_arttext

8. Sociedade Brasileira de Climatério. Consenso brasileiro multidisciplinar de assistência à mulher climatérica [Internet]. São Paulo (SP): SOBRAC; 2003 [atualizada em 05 de agosto de 2014; acesso em 23 de fevereiro de 2012]. Disponível em: http://pesquisa.bvsalud.org/portal/resource/ pt/lis-LISBR1.1-21461

9. Lorenzi DRS, Baracat EC. Climatério e qualidade de vida. Femina [Internet]. 2005 [acesso em 23 de fevereiro de 2012];33(12):899-903. Disponível em: http://bases.bireme.br/cgi-bin/wxislind.exe/iah/online/?lsisScript $=$ iah/iah. xis\&nextAction $=$ Ink\&base $=$ LILACS\&exprSearch $=4389$ 60\&indexSearch $=$ ID\&lang $=e$

10. Wood-Dauphinee S. Assessing quality of life in clinical research: from where have we come and where are we going? J Clin Epidemiol [Internet]. 1999 [cited 2012 February 23];52(4): 355-63. Available from: http://www.ncbi.nlm.nih.gov/pub $\mathrm{med} / 10235176$

11. Ciconelli RM. Tradução para o português e validação do questionário genérico de avaliação da qualidade de vida "Medical Outcomes Study 36 - item short-form health survey (SF-36)" [tese]. São Paulo (BR): Universidade Federal de São Paulo; 1997.
12. Heinemann LA, Potthoff $P$, Schneider HP. International versions of the Menopause Rating Scale (MRS). Health Qual Life Outcomes [Internet]. 2003 [cited 2012 February 23];(1):28-30. Available from: http://www.ncbi.nlm. nih.gov/pubmed/12914663

13. Heinemann LA, Ruebig A, Potthoff $P$, Hermann PG, Schneider HP, Strelow F, et al. The Menopause Rating Scale (MRS): a methodological review. Qual Life Outcomes [Internet]. 2004 [cited 2012 February 22];2:45. Available from: http://www.ncbi.nlm.nih.gov/pmc/articles/ PMC516787/

14. Sherman S. Natural history of menopause studies and related efforts at the National Institute on Aging, NIH. In: Schneider HPG, Naftolin F, editors. Climacteric medicine where do we go? London: Taylor \& Francis; 2005. p. 16-26.

15. O'dea I, Hunter MS, Anjos S. Life satisfaction and health-related quality of life (SF-36) of middle-aged men and women. Climacteric [Internet]. 1999 [cited 2012 February 22];2:131-40. Available from: http://www.ncbi.nlm. nih.gov/pubmed/11910666

16. Silva Filho EA, Costa AM. Avaliação da qualidade de vida de mulheres no climatério atendidas em hospital-escola na cidade do Recife, Brasil. Rev Bras Ginecol Obstet [Internet]. 2008 [acesso em 22 de fevereiro de 2012];30(3):113-20. Disponível em: http://www.scielo.br/scielo.php?pid = S0100-7 $2032008005000001 \&$ script $=$ sci arttext

17. Hays J, Ockene JK, Brunner RL, Kotchen JM, Manson JE, Patterson RE, et al. Effects of estrogen plus progestin on health-related quality of life. N Engl J Med [Internet]. 2003 [cited 2012 February 22]; 348(19):1839-54. Available from: http://www.ncbi.nlm.nih.gov/pubmed/12642637

18. Dennerstein L, Burrows GD, Hyman GJ, Sharpe K. Hormone Therapy and affect. Maturitas. 1979;1:247-59.

19. Marsh MS, Whitehead MI. Management of the menopause. Br Med Bull. 1992;48:426-57.

20. Wilson DH, Taylor AW, Maclennan AH. Health status of hormone replacement therapy users and non-users as determined by the SF-36 quality of life dimension. Climacteric. 1998;1(1):50-4.

21. Zahar SEV, Aldrighi JM, Pinto Neto AM, Conde DM, Zahar LO, Russomano F. Qualidade de vida em usuárias e não usuárias de terapia de reposição hormonal. Rev Assoc Med Bras [Internet].2005 [acesso 22 de fevereiro de 2012];51(3):133-8. 